\title{
Controlling Closed-Chain Robots with Compliant SMA Actuators: Algorithms and Experiments
}

\author{
Kyle Gilpin, Eduardo Torres-Jara, and Daniela Rus
}

\begin{abstract}
In this paper we present algorithms, devices, simulations, and experiments concerning a robot that locomotes using novel compliant, sheet-based, shape memory alloy actuators. Specifically, we describe the theory and practical implementation of a provably correct algorithm capable of generating locomotion gaits in closed-loop linkages. We implement this algorithm in a distributed fashion on the HexRoller, a closed-chain robot with six low-stiffness actuators. We describe these actuators in detail and characterize their performance along with that of the robot.
\end{abstract}

\section{Introduction}

We explore the design and control of robots with non-traditional mechanical properties such as high compliance whose motion is generated by programming their shape, stiffness, friction, or torque, for example. In particular, we are interested in affecting locomotion by changing the torque exerted by compliant joints in a closedchain robot such as that shown in Figure 1. Instead of using traditional motors which are rigid and heavy, we employ compliant shape memory alloy (SMA) actuators to serve as springs and exert torques. The innate compliance of these new SMA actuators ensures the system is mechanically robust. When inactive, the joints deform easily under light loads, but they can exert much larger torques when driven to their active state. By controlling the torque exerted by the joints, we simplify the control of complex closed linkages. A key technical challenge is designing appropriate actuators capable of this type of performance.

By applying torque to select joints of closed-chain robot with compliant joints, we can shift the center of mass of the robot to induce net forward motion. Each

Kyle Gilpin · Eduardo Torres-Jara · Daniela Rus

Distributed Robotics Lab, Massachusetts Institute of Technology

32 Vassar St, Cambridge, MA 02139 USA

e-mail: [kwgilpin|etorresj|rus] @csail.mit.edu 
step results in the robot's connection to the ground switching to a new face. By controlling the order in which we apply torque to the robot's joints, we show that a distributed controller can achieve continuous locomotion by rolling.

Using soft actuators capable of exerting torque for motion generation has several advantages over traditional motors. First, there is no need for careful coordination of the joints when executing parallel motions. Each joint can be controlled independently in a decentralized manner. The robot will roll forward when conditions on several joints are met, but these conditions do not have to initially arise at the same time. Second, there is no need to carefully tune control parameters. Third, our approach does not require precise constants as the robot's locomotion response can be characterized by a step function: below a certain torque threshold, the joint is too weak to generate any useful motion; above the threshold any torque value is sufficient (assuming we are operating on a horizontal plane). Finally, due to the redundancy associated with many joints in a closed chain, the system should accommodate joint failures without affecting functionality.

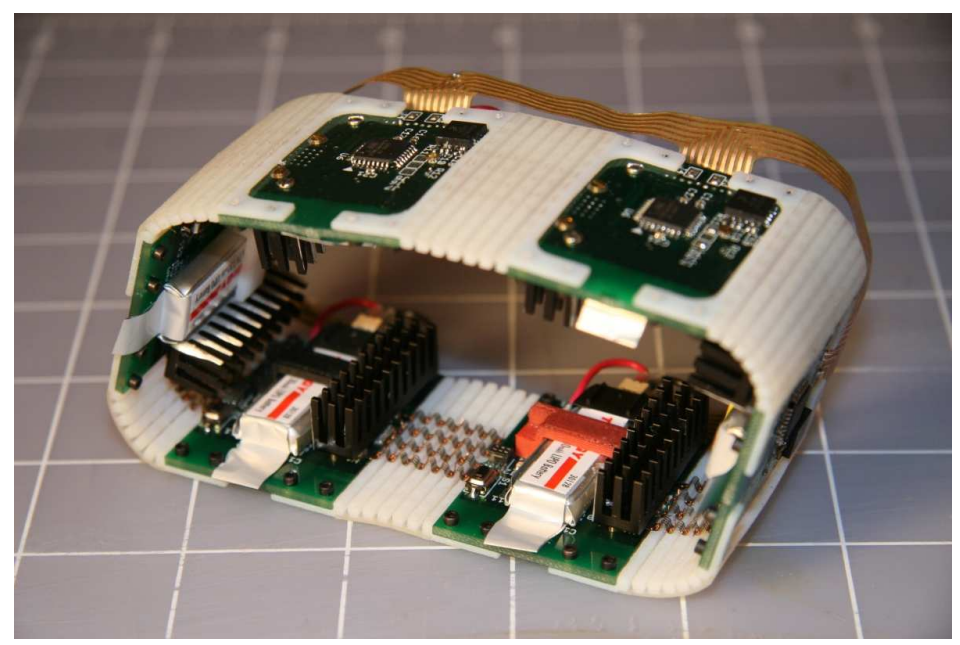

Fig. 1 The HexRoller is a highly compliant robot constructed from six circuit boards joined by SMA-driven flexible actuators. The robot is self-powered and completely autonomous.

In this paper we present algorithms, devices, and experimental results with a robot that locomotes using our soft SMA actuators. Specifically, we describe the theory and practical implementation of a generic algorithm for generating locomotion gaits in closed-loop linkages. This provably correct algorithm can be applied to linkage robots with an arbitrary number of identical links connected by compliant joints. We also present hardware for the HexRoller linkage robot shown in Figure 1 that can move using our locomotion algorithm. The key innovation associated with the HexRoller is the novel actuator shown in Figure 6. It is light-weight but capable of exerting non-trivial torques. We describe and characterize this actuator, and then we implement a distributed version of the locomotion algorithm on the HexRoller. 


\subsection{Related Work}

Closed-chain robots initially grew out of the larger modular robotics field. Yim developed one of the earliest examples of a rolling robot chain using his 2-DOF Polypod modules [17]. Kamimura et al. [7] employed central pattern generators to realize rolling locomotion gaits in the MTRAN system. Shen et al. have also simulated a loop of six SuperBot modules implementing efficient rollings gaits [13]. To date, Yim et al. [12] claim to have created one of the fastest modular rolling robots by incorporating position feedback into a control loop driving a closed chain of CKBot modules. In other work, Yim et al. [10] have rigorously analyzed what types of elliptical shapes can produce rolling locomotion. Matsuda et al. [9] have developed a rolling chain robot with variable stiffness joints. These joints employ a leaf spring with variable hinge points to transmit force from a traditional motor to the actuator. This method is commonly used to to make a traditional stiff actuator present a lower mechanical impedance [11] at the mechanism's output. However, the underlying actuator is still rigid.

In our work, we consider soft mechanisms in which all constituent parts exhibit low mechanical impedance. The actuator used is based on shape memory alloy (SMA) sheets and a flexible support structure. Other authors have proposed a number of ways to employ SMA (wire or sheet) as soft actuators. For example, SMA has been used to actuate a number of different robotic devices: hands [3], a biped [6], a micro-robotic fish [4], worms [8], and rollers [14]. However, the actuator that we use displays features that are useful in our particular application. For instance, the actuator is power-efficient and has an excellent strength to weight ratio [16] enabling our entire system to carry its own battery on board.

\section{Conceptual Algorithms}

Our goal was to design a robust algorithm that leverages compliant joints in a closedloop linkage to simplify the sensing, planning, and computation required in order to achieve locomotion through rolling. To simplify the initial design and analysis, we focused on a closed, planar, chain-type linkage capable of rolling in a straight line. All joints in the chain have some stiffness, and the algorithm can program each joint to exert additional torque on its adjacent links. The algorithm relies on identifying what we term the grounded link, grounded joint, leading link, and leading joint as shown in Figure 2. The grounded link is the link that is nearest the direction of motion while still resting completely on the ground. Similarly, the grounded joint is the joint connected to the grounded link nearest the direction of motion. The leading link is the other edge, (the one not resting on the ground), connected to the grounded joint. Finally, the leading joint is the other joint attached to the leading link.

The basic idea behind the algorithm is to shift the projection, (onto the ground), of the center of gravity of the entire structure sufficiently far in the direction of motion that the structure changes on which links it is resting, in essence "falling" onto 

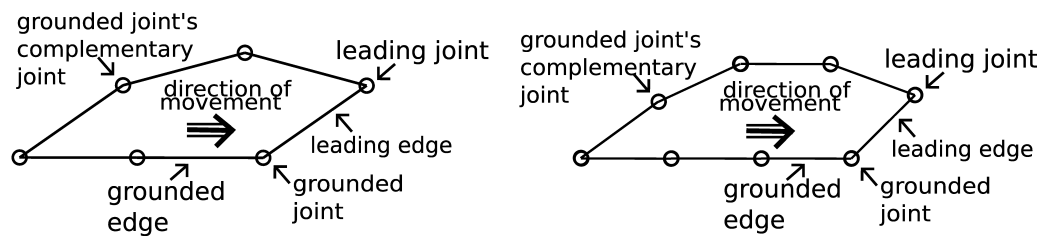

Fig. 2 With respect to the direction of motion, the grounded joint is the forward-most joint that is still on the ground. It connects the grounded edge (behind) to the leading edge (in front). The other joint attached to the leading edge is the leading joint.

the leading edge. To achieve this shift in the center of gravity, the algorithm makes a choice based on the structure's nominal stiffness and weight. We characterize the stiffness of the structure as either "high" or "low" based on whether the linkage structure has two or more faces in contact with the ground. If the structure has multiple faces in contact with the ground, (see Figure 3), it is deemed low stiffness as the joints are not stiff enough to support the structure's weight. If the structure is stiff enough that only one face is in contact with the ground, (see Figure 4), it is a high stiffness structure.

If the structure is low stiffness, the algorithm applies torque to the grounded joint, shown in red in Figure 3. This creates an asymmetry about the vertical mid-line of the structure. With enough torque, the leading linkage is drawn downward pulling the other links in the structure forward. As the grounded joint applies more torque, this asymmetry can be increased arbitrarily. This forward motion is aided by the fact that, in practice, the joints have inherent mechanical limits. As the center of gravity shifts forward, the leading joint and its complement cannot collapse completely forcing the other joints to reconfigure and the trailing linkage to lift from the ground. As a result, the projection of the center of gravity will shift past the midpoint of the grounded link, and the structure will come to rest on its leading edge.

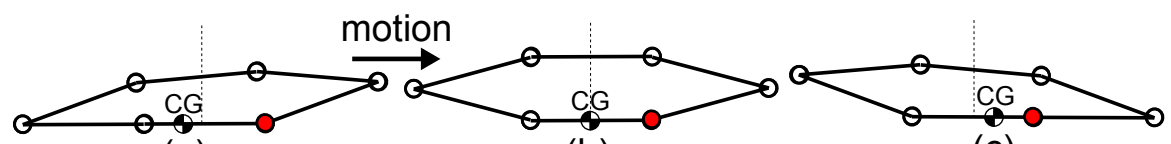

(a)

(b)

(c)

Fig. 3 The low stiffness rolling sequence begins when the projected center of gravity is on the far left side of the center line of the grounded link(a). As the grounded joint (red) is activated, the projected center of gravity shifts past the center line (b) and comes to rest on the near side (c) as the leading edge comes to rest on the ground.

In the case of a high stiffness structure, the algorithm must actuate both the grounded joint and the leading joint. As shown in Figure 4(a-c), if the algorithm 
only actuates the grounded joint (red), it will pull the leading edge down parallel to the ground, and the projection of the center of gravity will asymptotically approach the grounded joint. But, due to the equal stiffness of all other joints and the symmetric structure that results, the center of gravity will never pass over the grounded joint. As shown in Figure 4(c), the structure has been effectively reduced to the low stiffness case, (two edges in contact with the ground). Now, to initiate rolling, one must actuate what was the leading joint (blue), to induce the asymmetry necessary to shift the center of gravity past the grounded joint. In practice, the grounded and leading joints can be actuated together to quicken the process.

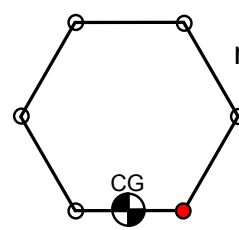

(a)

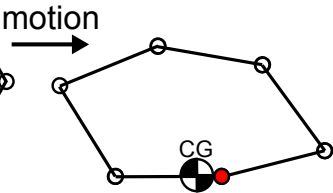

(b)

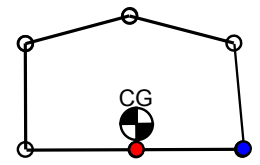

(c)

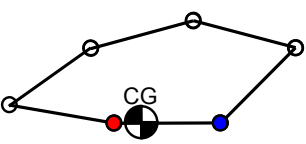

(d)

Fig. 4 For high stiffness structures, the rolling sequence can be decomposed into two steps. From rest (a), the grounded joint (red) is actuated and the projection of the center of gravity shifts toward it (b). Even when the stiffness of the grounded joint is infinitely large, the center of gravity will not shift past the grounded joint (c). Only by also energizing the leading joint (blue) in frame (d) can we induce the asymmetry needed to shift the center of gravity past the red joint to induce rolling.

Once the structure has rolled, the applied torques are removed, and the process may be repeated. The added torque necessary to induce rolling may be reduced in even-sided structures by employing complementary joints. A joint's complement is the joint on the exact opposite side of the structure. In a hexagon whose joints are numbered sequentially, the following are pairs of complementary joints: $(1,3),(2,4)$ and $(3,6)$. By actuating the complementary joints in parallel with the primary joints, one reduces the torque required of each joint. This arises from the symmetry of the structure: where as the grounded and leading joints pull the structure to one side, their complementary joints push by the same amount.

\section{Proof of Concept Simulations}

Analytical descriptions of mechanical linkages have been well studied [5], but their complexity as the number of linkages grows drives us to work with numerical simulations instead. Additionally, our system contains closed chains and highly nonlinear constraints such as hard joint limits that make the modeling problem particularly intractable. We created a simulator based on Open Dynamics Engine (ODE) [2] physics simulator to test our control algorithms. In addition to modeling the stiffness of the joints, ODE models gravity and the mass of the links connecting the joints. The simulator, through the use of configuration files, allows us to control almost 
any aspect of the linkage being simulated. We can set the stiffness of the joints, their damping constants, the mass of the faces of the closed-loop linkage, their size, and the physical parameters of the joints, for example. Using the mouse while the simulation is running, one can select specific joints to which he wants to apply additional torque. This allows us to estimate, for a given model, how much torque the actuators need to apply to induce locomotion.

For the purposes of this paper, we focused on simulating a 2D hexagon similar to the HexRoller hardware. We correctly model its size, weight, and nominal joint stiffness. The only major departure from the physical hardware is that the joints in the simulation are not continuously deformable. In the simplest case, the joints in the simulation are point hinges. Given that the joints in the HexRoller are $17.0 \mathrm{~mm}$ long and the links are only $30.0 \mathrm{~mm}$ long, we were concerned that this was not an accurate model, so we compared the point hinge case to models with two and three sub-hinges per joint. (We would have liked to continue with higher dimensional cases, but the simulator became unstable when trying to simulate four or more subhinges per joint.) The results of our comparison are shown in Table 1. In each of the three cases, the total weight of the structure and the length of the faces was unchanged. In the two and three sub-hinge cases, the weight of the joints was placed in the mechanical structures that connected the sub-hinges. In the point hinge case, the weight of the joint was incorporated into the faces because the sub-hinge connectors did not exist. The results do indicate that modeling the joint correctly is important. As the simulated joint approaches a continuously flexible surface, the torque required to induce locomotion decreases. Given that we cannot simulate more than three sub-hinges, we expect the simulator to overestimate the required torques.

Table 1 The number of sub-hinges used to model the flexures found in the HexRollers joints effects how much torque the simulator predicts is required to induce locomotion if both the grounded joint and its complement are actuated.

\begin{tabular}{|c|c|}
\hline Number of Sub-hinges & Min. Torque to Induce Rolling [mNm] \\
\hline \hline 1 & 5.3 \\
\hline 2 & 3.0 \\
\hline 3 & 2.4 \\
\hline
\end{tabular}

Using the simulator, we were able to verify the correctness of the above approach to locomotion and estimate the torque requirements of each joint. The amount of torque the joints must exert to roll the structure depends on the nominal stiffness of the joints. This is illustrated in Figure 5 which indicates there is an optimal nominal stiffness (b) which minimizes the required joint torque. Below the optimal stiffness, the required torque grows slowly. In this region, two or more faces of the roller are firmly planted on the ground. Above the optimal stiffness, the roller only rests on one face, and the torque required for locomotion grows rapidly. The simulations were performed with a simplified joint model comprised of a point hinge. As indicated in Table 1, the actual torque required as the hinge begins to approximate a continuous flexure will be lower. 

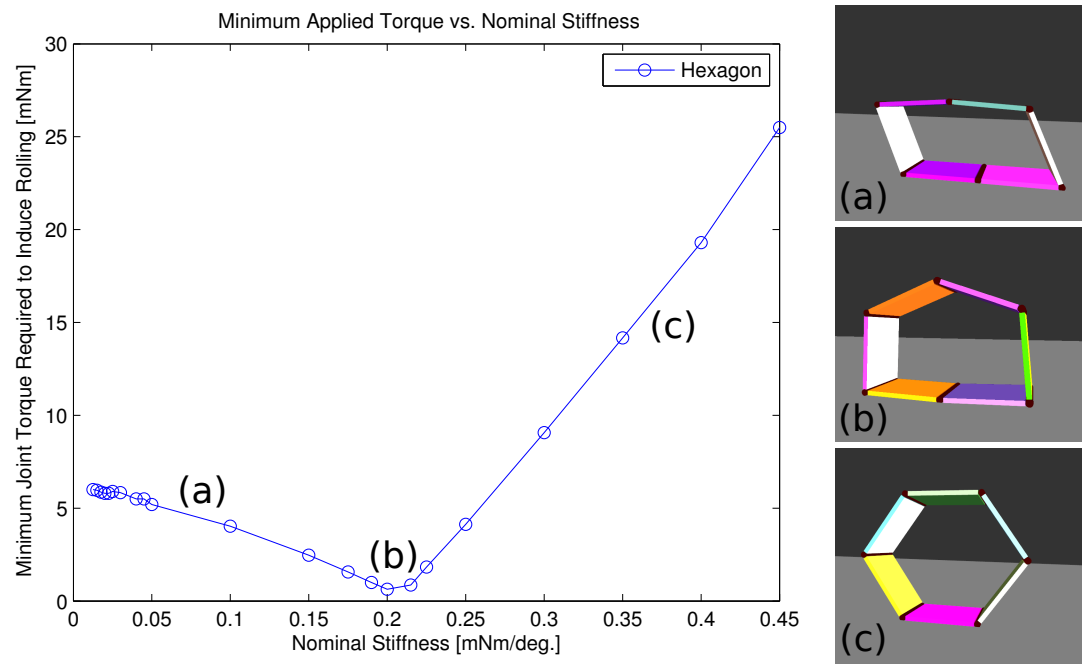

Fig. 5 The nominal stiffness of each joint in a closed-chain rolling robot affects the torque that must be exerted by the joints in order to induce rolling. The different stiffness regimes $(\mathrm{a}, \mathrm{b}, \mathrm{c})$ marked in the graph correspond to the nominal stiffness scenarios shown on the right. In the optimal regime, (b), the roller is just barely balancing on a single face. Note: we always applied torque to two joints at a time: the grounded joint and its complement in the low stiffness case and the grounded and leading joints in the high stiffness case.

\section{Hardware: The HexRoller and its Actuators}

In order to demonstrate the correct of our algorithms, we built the HexRoller, shown in Figures 1 and 6. Each module of the chain is formed by a $30 \mathrm{~mm}$ long, by $40 \mathrm{~mm}$ wide, PCB attached to a flexible shape memory alloy (SMA) actuator. The SMA actuator attached to each PCB adds an additional $18 \mathrm{~mm}$ to the length of the module. Each module contains an Atmel microprocessor, SMA driver circuitry, a 2-axis accelerometer, and a rechargeable lithium polymer battery allowing the system to operate completely autonomously.

The modules are light weight: the populated PCB, SMA driver heat sink, battery, and battery holder weigh $5.13 \mathrm{~g}, 2.5 \mathrm{~g}, 3.56 \mathrm{~g}$, and $0.35 \mathrm{~g}$, respectively. The SMA actuators attached to each PCB weigh $2.36 \mathrm{~g}$. When fully assembled, the entire sixmodule HexRoller weighs $89.7 \mathrm{~g}$. The design is completely modular allowing quick replacement or the addition of extra modules. Any number of modules can be fitted with a wireless transceiver [1] in order to communicate with a PC base station. The modules communicate with their neighbors using a shared two-wire bus that is routed through a flex circuit running around the perimeter of the robot. The bus operates as a multi-master system. The flex circuit also carries power lines between the modules. While a battery is attached to each module, the entirety of the voltage and current required by the SMA actuators cannot be supplied by a single cell. As a 


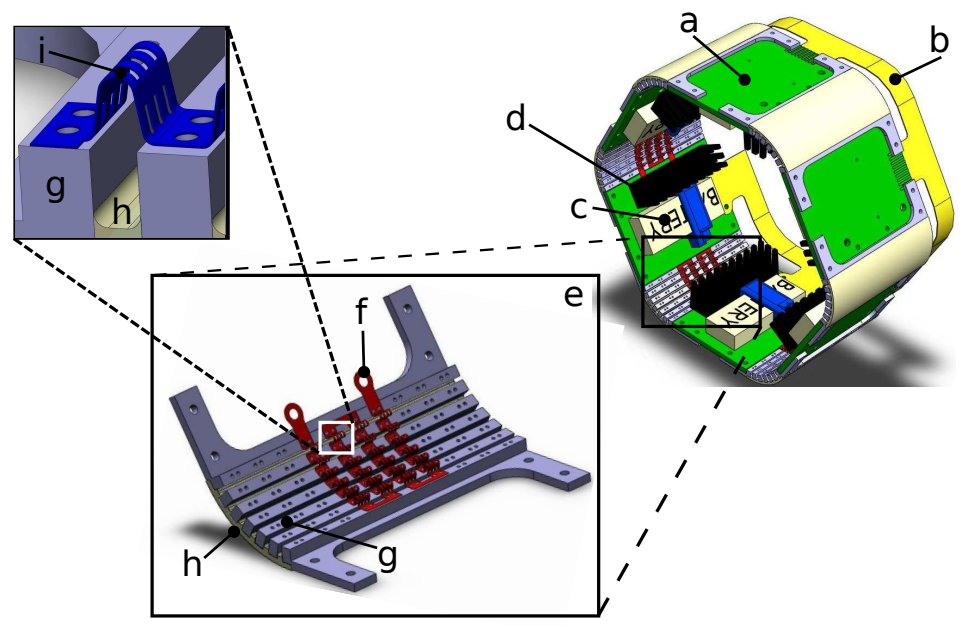

Fig. 6 The HexRoller consists of six PCBs (a) that are electrically connected with a flexible circuit (b) that carries communication signals and distributes the power load among the six batteries (c). Each module contains driver circuitry and a heat sink (d) to control an SMA actuator (e). The actuators are composed of a laser-cut SMA sheet (f) mounted on rigid supports ( $g$ ) that are embedded in a flexible substrate (h). Each actuator is composed of 24 unit cells (i).

result, the batteries are wired into there parallel groups; each group containing two batteries in series for a total net rating of $390 \mathrm{mAh}$ at $7.4 \mathrm{~V}$.

\subsection{SMA Actuators}

Alternatives to traditional actuators such as electromagnetic motors are needed in applications where space and weight are limited. We have developed a family of small, lightweight actuators [16, 15] based on Shape Memory Alloy (SMA) sheets. SMA is a material that recovers its original shape when heat is applied. In particular, heat causes SMA to transition from its martensitic to austenitic phase. During this phase change, the SMA exerts force in an effort to return to its annealed shape. If a flat sheet of SMA is deformed, heating the sheet will cause it to flatten. Using this principle, we designed an actuation unit cell that is labeled (i) in Figure 6. In this design, the central crease of $180^{\circ}$ and the peripheral creases of $90^{\circ}$ will all exert torques when the structure is heated. These torques tend to unbend the SMA sheet. The smaller the radius of the bend, the greater the torque that will be exerted. By attaching this unit cell to a flexible substrate, (Figure 6h), we can redirect this torque to produce a macroscopic folding motion. Changing the spacing of the creases allows us to tailor the peak force, the bend angle at which the peak force is exerted, and the total travel distance. 
To create a more versatile actuator, we combined many of these unit cells into a larger structure. For the HexRoller, we built the actuator shown in Figure 6e by combining 24 units of a basic actuation cell (Figure 6i). The cells are arranged in four parallel columns of six cells each. Each section is divided by a support element where the cells are attached to a rigid anchor bar that is embedded in the flexible substrate. This composite support structure is produced through a 3D printing process on an Objet Connex printer. The printer can manufacture the entire assembly in one pass, depositing both the rigid and flexible materials simultaneously. The flexible material is $0.51 \mathrm{~mm}$ thick. The $1.50 \mathrm{~mm}$-wide rigid support bars extend out of the flexible material $1.65 \mathrm{~mm}$. The SMA piece is attached to the support bars with $0.5 \mathrm{~mm}$ diameter screws or through sewing with \#32 AWG copper wire.

Our design overcomes the traditional limitations present in actuators based on SMA wires. In our design, the heat needed to activate the SMA is focused in a localized area. This is accomplished by laser cutting the flat SMA sheet with a specific pattern that creates high resistance pathways. These high resistance areas are aligned with the creases in the unit cells so that the creases heat up while the surrounding material remains relatively cool. This is an important consideration given that the SMA needs to reach $95^{\circ} \mathrm{C}$. By controlling the points of heat generation, we reduce unnecessary heating, and the unheated areas can be used to mount the actuator. Additionally, the SMA sheet is mounted in such a way that it is difficult to over-strain the SMA material. In other applications, a strain exceeding 5\% can ruin the SMA's ability to return to its annealed shape when heated. By mounting our SMA actuator on a flexible substrate that constraints its movement, we prevent damage from overstrain. Finally, the total cross-sectional area of our sheet-based actuators is large, allowing us to exert significant forces with a relatively small, lightweight device. The actuator is $17.0 \mathrm{~mm}$ long, $40.0 \mathrm{~mm}$ wide, $2.85 \mathrm{~mm}$ tall, and it weighs only $2.36 \mathrm{~g}$. When the actuator is energized, it can exert $3.0 \mathrm{mNm}$. A more detailed evaluation of the torque generated by the actuator is presented in Section 5

Each module drives its attached SMA actuator with a low noise linear regulator. While not optimally efficient, it generates little noise. As a result, each module can precisely monitor and control the current flowing through its SMA. By also measuring the voltage dropped across the actuator, the module can determine the actuator's resistance. As shown in Section 5, the actuator's resistance can be employed to control it more efficiently.

\section{Experimental Results}

We performed a number of experiments on the individual SMA actuators and the HexRoller robot. These experiments characterize the electrical and mechanical response of the actuators. The experiments with the HexRoller illustrate that the robot operates as expected and is capable of achieving locomotion using our actuators. 


\subsection{SMA Actuators}

In order to determine the electrical response of the actuator, we applied a constant current and measured the voltage drop. In the first set of experiments, we applied $1.5 \mathrm{~A}$ and recorded the resistance of the actuator as shown on the left in Figure 7. When current is first applied, the resistance increases. This makes intuitive sense given that there is typically a positive correlation between temperature and resistance in metals. After additional heating, the SMA reaches its transition temperature, and as the SMA changes from its martensitic to austenitic phase, the resistance drops approximately $10 \%$ in less than $100 \mathrm{~ms}$. We also measured the response time of the actuator to other values of electrical current. These times are shown on the right of Figure 7 where we observe the same drop in resistance as the SMA reaches its transition temperature. The intensity of the electrical current inversely affects the time taken to reach the transition temperature.
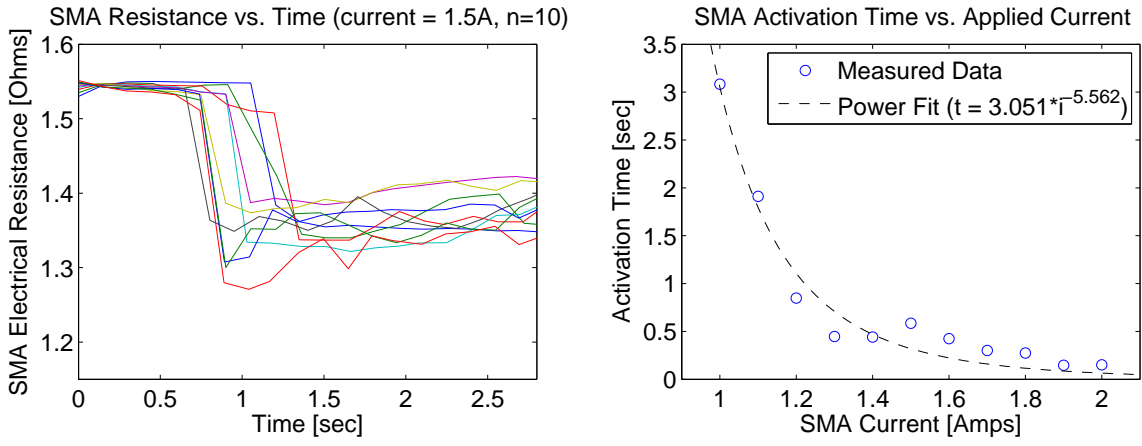

Fig. 7 The left sub-figure illustrates that when 1.5A is applied to a cool actuator, the resistance of the SMA increases steadily until the SMA's transition temperature is reached. At this point, there is a sudden $10 \%$ drop in the resistance as the SMA begins recover its annealed shape. In the right sub-figure, the current applied to the SMA was swept from 1.0A to $2.0 \mathrm{~A}$ in order to determine the response time of the SMA actuator as indicated by a change in its resistance.

We determined the torque response of the actuator using the setup shown in Figure 8 . Instead of measuring the torque directly, we used a load cell, motion stage, and accelerometer (to determine angle $\theta$ ), to calculate the torque exerted by the actuator. Unfortunately, we could not account for the SMA bending with nonuniform curvature. Results from measuring the actuator's torque when passive and energized are shown in Figure 9.

As illustrated in the plots, the actuator behaves as a spring, exerting a torque that is proportion to its deviation from $\theta_{0}$, its rest angle. Using the unactuated data, we compute that $\theta_{0}=60.4^{\circ}$ where $0^{\circ}$ corresponds to a flattened actuator, and positive angles indicate that the flexible substrate is bending away from the SMA-side of the actuator. When the actuator is energized, it exerts an additional torque of $0.94 \mathrm{mNm}$. The spring constant also changes when it is actuated, but the additional force due 


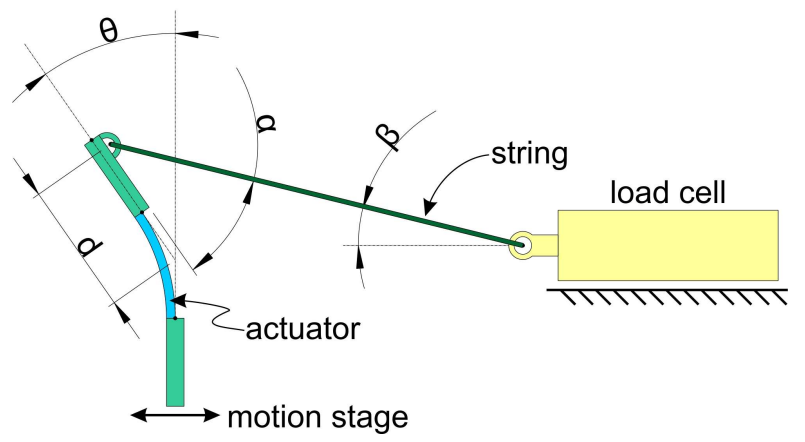

Fig. 8 A load cell was used to estimate the torque exerted by the actuator through a lever arm of length $d$ as a function of the deformation angle $\theta$. The actuator was anchored to a precision motion table so that $\theta$ could be varied. The other end of the actuator was anchored by a string to the load cell on the right. In this setup, the SMA faces the load cell, and the indicated $\theta$ is positive.

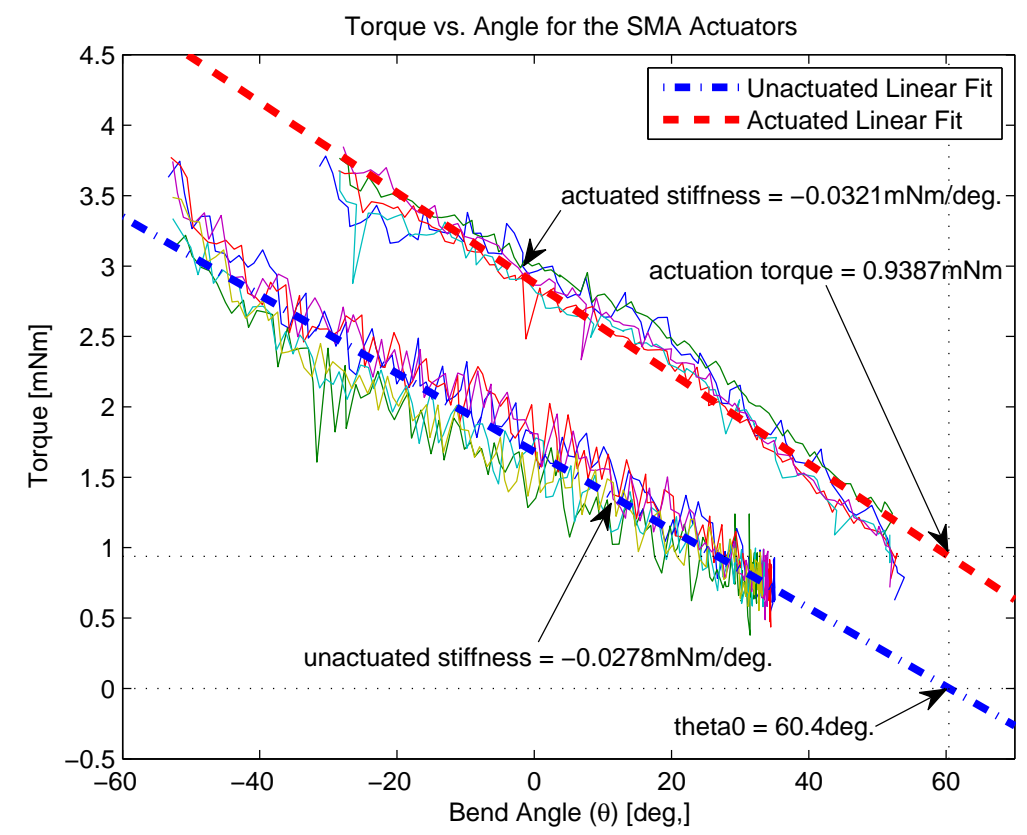

Fig. 9 When the SMA actuator is off, it approximates a spring-the torque it exerts is proportional to the angle through which it is bent. The actuator's resting position is $\theta=60^{\circ}$ (with the flexible substrate curving away from side on which the SMA is attached). When the SMA actuator is energized, its exerts an additional $0.9387 \mathrm{mNm}$ of torque, and the spring constant increases by $15 \%$. 
to the new spring constant does not reach parity with the additional torque until the actuator is deformed to $-218^{\circ}$, an impossibly large angle.

Having characterized the actuators, it is interesting to compare their torque output with the prediction, made by the simulator, of the torque necessary to achieve rolling locomotion. The simulator predicts a torque of $2.4 \mathrm{mNm}$ is necessary to induce rolling, through, as addressed in the discussion of Table 1 above, this estimate is too high. At $\theta_{0}$, the SMA actuators exert only $0.94 \mathrm{mNm}$, but if one takes into account the increased spring constant of the energized actuators and the fact that they operate about $\theta=-70^{\circ}$, the actuators actually contribute $1.50 \mathrm{mNm}-\mathrm{a}$ torque within $60 \%$ of the admittedly high prediction. Regardless of the simulator's predictions and the extrapolated stiffness curves, he HexRoller works as intended.

\subsection{HexRoller}

The initial test of the HexRoller was accomplished with an open-loop controller running on a PC wirelessly sending commands to one master module from where they were distributed to the other modules in the chain. The control program executing on the PC is provided with the HexRoller's initial position. From that, it produces a set of commands at fixed intervals to energize the leading joint and its complementary joint in the correct sequence as described in Section 2. The actuators are energized for long enough time periods to ensure that there is sufficient time to roll from one face to the next. There is no feedback to the controller, so if the HexRoller misses a step, the subsequent commands will be incorrect until the controller resynchronizes with the hardware. Using the open-loop controller, the HexRoller moved $30.48 \mathrm{~cm}$ in $22.67 \mathrm{~s}$ for a speed of $1.3 \mathrm{~cm} / \mathrm{s}$. The HexRoller rolled 7 times during this period, or once roll every 3.23 seconds. One "step" is shown in Figure 10.

\subsection{Distributed Algorithm}

We also implemented the closed-loop distributed algorithm shown in Algorithm 1 which is capable of controlling the HexRoller more efficiently than the open-loop controller. The algorithm works using only the module's orientation, and the orientations of its two closest neighbors. Modules share their orientation information with their neighbors over the two-wire bus every time their orientation changes. The control loop runs at $10 \mathrm{~Hz}$ constantly checking the orientation of the module and its two neighbors. As soon as the module detects that its actuator is positioned in the grounded joint position or its complement, it energizes the actuator with $1.5 \mathrm{~A}$. The actuator remains energized until the module detects that it is no longer the grounded joint or its complement. As a result, as soon as the structure rolls onto the leading face, the old grounded joint and its complement turn off and the new grounded joint and its complement turn on. There is an additional safety feature built in to the al- 

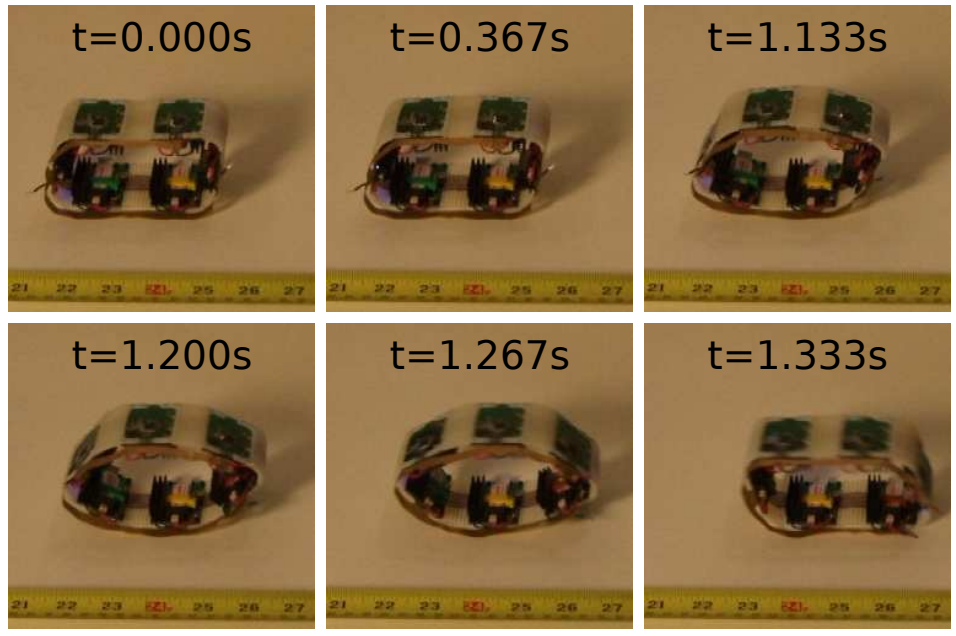

Fig. 10 The critical section of one step of the open-loop rolling sequence takes $1.3 \mathrm{~s}$. This sequence shows the most significant events starting from a reference point that we designated $t=0.000 \mathrm{~s}$

gorithm that forces the SMA actuator off if it has been energized for more than 4 seconds. The actuator is allowed to cool for 10 seconds before it can be reactivated. This ensures that the actuators do not overheat and destroy themselves.

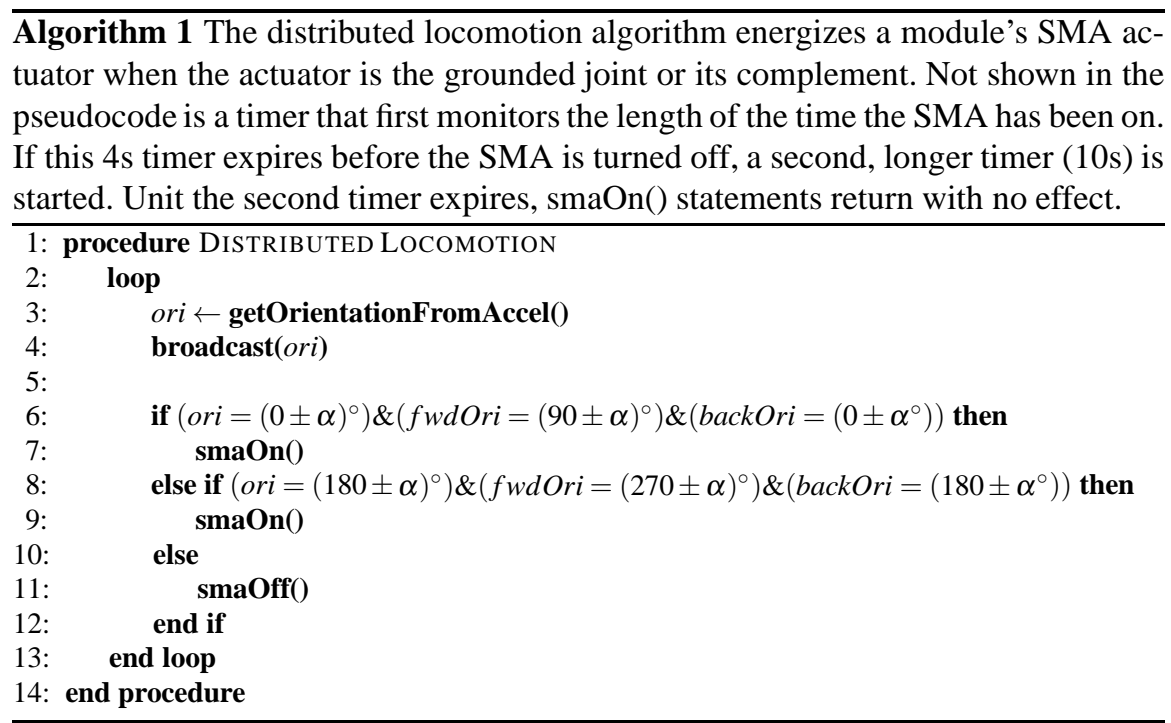

Using the closed-loop distributed controller, the HexRoller moved $27.94 \mathrm{~cm}$ in $9 \mathrm{sec}$ for a speed of $3.1 \mathrm{~cm} / \mathrm{sec}$. The speed of the closed-loop distributed controller is more than twice that of the open-loop centralized controller. We also tested the 
reliability of the distributed algorithm and hardware by allowing the HexRoller to step until it failed. In five tests, the robot was able to take $32,12,16,16$, and 48 steps respectively. During all five runs, the robot only misstepped a total of 7 times, each time recovering on its own. It should be noted that the 48 step test was performed immediately after charging the robot's batteries. At the end of this test, the performance degradation was noticeable. In part, we believe that the batteries were depleted. Additionally, we suspect that after 48 steps, the average actuator temperature has risen to a point where the actuators did not have time to adequately cool between cycles. Finally, some of the failures of the robot can be attributed to the inability of the processors to recover from certain types of collisions on the two wire inter-processor bus. When these collisions occur, one or more of the processors locks up and stops actuating its joint. In three of the cases were the HexRoller stopped moving, one of the processor was later found to have locked. This could be rectified by employing the processor's watchdog timer.

\section{Discussion}

The HexRoller robot is still not as functional as we envision that it could be. First, the HexRoller can only move in one dimension. In the future, we plan on connecting two or three HexRollers at orthogonal angles to form a robot resembling a sphere. Alternatively, we have considered arranging four HexRollers in a square, (the long edges of their faces coincident and the faces at right-angles), to form a "QuadHex." Another possibility is to split the actuators along their longitudinal axes so that the two sides may contract independently to generate curved trajectories.

The current instantiation of the HexRoller is not as efficient as it could be. The linear regulator powering the SMA could be replaced with a switching topology. Likewise, the resistance sensing capability could be employed as the input to a feedback loop that both speeds-up the SMA response and reduces the SMA power dissipation to a steady-state level once the actuator has reached its transition temperature. The results of the simulations above also indicate that by increasing the stiffness of the joints to an optimum value we could reduce the demands on the actuators.

Future work can also improve the control algorithms. The current advantage to our approach is the simplicity, but we believe that improvements can be made without sacrificing too much. For one, the update rate of the controller should be increased so that the system responds more quickly to changes in orientation. Second, the processors in the current distributed algorithm loose synchronization if they do not detect their relative positions in the loop simultaneously. If one actuator is not strong enough to roll the loop, it will waste power waiting for the other to turn on.

This paper also points to a number of important open problems. From a theoretical point of view, we need to rigorously model the complex physical interactions between the SMA actuators in a closed chain. We would also like to develop an algorithm that will generate locomotion gaits for arbitrary closed chain linkage structures. While not complete, the work presented in this paper can be viewed as a 
small first step toward controlling machines with a non-traditional mechanical properties, such as low-stiffness actuators, which have many advantages over traditional, high-stiffness alternatives composed of motors and gears.

\section{Acknowledgments}

This work is supported by the DARPA Programmable Matter program and the US Army Research Office under grant number W911NF-08-1-0228, and the NDSEG fellowship program. The authors would also like to thank Josh Karges for his work preparing many of the figures and building the prototypes.

\section{References}

1. Nordic semiconductor, inc. http://www. nordicsemi.com/ (2010)

2. Open dynamics engine. http://www.ode.org/ (2010)

3. Cho, K.J., Asada, H.: Multi-axis sma actuator array for driving anthropomorphic robot hand. In: ICRA, pp. 1356-1361 (2005)

4. Cho, K.J., Hawkes, E., Quinn, C., Wood, R.: Design, fabrication and analysis of a body-caudal fin propulsion system for a microrobotic fish. In: ICRA, pp. 706-711 (2008)

5. Craig, J.J.: Introduction to Robotics: Mechanics and Control. Addison-Wesley (1989)

6. Esfahani, E., Elahinia, M.: Stable walking pattern for an sma-actuated biped. Mechatronics 12(5), 534-541 (2007)

7. Kamimura, A., Kurokawa, H., Yoshida, E., Murata, S., Tomita, K., Kokaji, S.: Automatic locomotion design and experiments for a modular robotic system. IEEE/ASME Transactions on Mechatronics 10(3), 314-325 (2005)

8. Kim, S., Hawkes, E., Cho, K.J., Joldaz, M., Foley, J., Wood, R.: Micro artificial muscle fiber using niti spring for soft robotics. In: IROS, pp. 2228-2234 (2009)

9. Matsuda, T., Murata, S.: Stiffness distribution control-locomotion of closed link robot with mechanical softness. In: ICRA, pp. 1491-1498 (2006)

10. Mellinger, D., Kumar, V., Yim, M.: Control of locomotion with shape-changing wheels. In: ICRA, pp. 1750-1755 (2009)

11. Pratt, G., Williamson, M.: Series elastic actuators. In: IROS, pp. 399-406 (1995)

12. Sastra, J., Chitta, S., Yim, M.: Dynamic rolling for a modular loop robot. International Journal of Robotics Research (IJRR) 28(6), 758-773 (2009)

13. Shen, W.M., Krivokon, M., Chu, H., Everist, J., Rubenstein, M., Venkatesh, J.: Multimode locomotion via superbot reconfigurable robots. Autnomous Robots 20(2), 126-177 (2006)

14. Sugiyama, Y., Shitsu, A., Yamanaka, M., Hirai, S.: Circular/spherical robots for crawling and jumping. In: ICRA, pp. 3595-3600 (2005)

15. Torres-Jara, E., Gilpin, K., Karges, J., Wood, R.J., Rus, D.: Composable flexible small actuators built from thin shape memory alloy sheets. Robotics and Automation Magazine (In press)

16. Torres-Jara, E., Rus, D.: Flexible actuator based on shape memory alloy sheet (us patent 61/248,83) (2009)

17. Yim, M.: New locomotion gaits. In: IEEE International Conference on Robotics and Automation (ICRA), pp. 2508-2514 (1994) 